\title{
Le datif éthique : entre écriture et oralité
}

\author{
Elena Puerta Moreno \\ Profesora Sustituta Interina \\ Universidad de Sevilla \\ C/Palos de la Frontera \\ E-mail: epmoreno@us.es \\ Tel.: 664883493
}

\section{LE DATIF ÉTHIQUE : ENTRE ÉCRI- TURE ET ORALITÉ}

RÉSUMÉ : Utilisé intuitivement par les locuteurs, le datif éthique, un pronom non envisagé dans la structure verbale et qui dépasse d'ailleurs les limites de la syntaxe, devient une marque d'oralité pour caractériser le discours direct dans les textes écrits, notamment littéraires. Nous proposons donc que sa définition comme élément commun entre l'oralité et l'écriture passe actuellement par l'étude de son usage sur les réseaux sociaux, ainsi que sur les sites Internet repérés par l'application Sketch Engin, dans la mesure où ces deux supports donnent lieu à un type d'écriture conditionnée par la spontanéité inhérente au discours oral.

MOTS CLÉS : datif éthique ; réseaux sociaux ; écrit et oral ; grammaire contrastive ; linguistique de corpus.

SOMMAIRE : 1 . Introduction. 2. Le datif éthique. 3. Entre écriture et oralité : le cas d'Internet et des réseaux sociaux. 4. Conclusions.
THE ETHICAL DATIVE: BETWEEN WRITING AND SPEAKING

ABSTRACT: Used intuitively by speakers, the ethical dative, a pronoun not contemplated in the verbal structure that goes beyond the limits of syntax, has often become a mark of orality to characterize direct discourse in written texts, especially literary ones. Therefore, we propose that its definition as a shared element between orality and writing should currently be analysed through the study of its use in social networks, as well as in some websites taken up by the Sketch Engine application, insofar, as both supports give rise to a type of writing conditioned by the inherent spontaneity of oral discourse.

KEY WORDS: ethical dative; social networks; writing and oral; contrastive grammar; corpus linguistics.

SUMMARY: 1 . Introduction. 2 . The ethical dative. 3. Between writing and speaking: the case of the Internet and social networks. 4 Conclusions.
EL DATIVO ÉTICO: ENTRE ESCRITURA Y ORALIDAD

RESUMEN: Utilizado de manera intuitiva por los hablantes, el dativo ético, pronombre no contemplado en la estructura verbal que sobrepasa los limites de la sintaxis, se ha convertido en numerosas ocasiones en una marca de oralidad para caracterizar el discurso directo en los textos escritos, especialmente literarios. Por lo tanto, planteamos que su definición como elemento compartido entre oralidad y escritura debe pasar actualmente por el estudio de su empleo en las redes sociales, así como en ciertas páginas de internet retomadas por la aplicación Sketch Engine en la medida en la que ambos soportes suscitan un tipo de escritura condicionada por la espontaneidad propia al discurso oral.

PALABRAS ClAVes: dativo ético; redes sociales; escritura y oralidad gramática contrastiva; lingüistica de corpus.

SUMARIO: 1. Introducción. 2. El dativo ético. 3. Entre escritura y oralidad: el caso de Internet y de las redes sociales. 4. Conclusiones. 


\section{Le datif éthique : entre écriture et oralité}

Elena PUERTA MORENO

\section{INTRODUCTION}

Le datif éthique, pronom utilisé intuitivement par les locuteurs, reste cependant en marge des études de grammaire appliquée. Encore assez méconnu, car peu d'études approfondissent son usage et morphologie, notamment en espagnol où la bibliographie est plus réduite, ce type de datif échappe à la norme et aux règles grammaticales. Un élément grammatical séduisant d'autant plus qu'il dépasse les rapports syntaxiques pour s'inscrire dans le domaine de l'énonciation, voire de la pragmatique.

Utilisé dans nos langues d'étude (français et espagnol) quoique chacune déterminée par ses particularités ${ }^{1}$ comme nous verrons par la suite, ce pronom partage une même origine commune : le cas datif du latin. Ce dernier, caractérisé par l'expression d'une relation spécifique entre les compléments, n'est pas forcément déterminé par le verbe, comme dans le cas du datif éthique qui ne fait qu'apporter des nuances sémantiques aux énoncés sans être obligatoire pour la réalisation du verbe (Rubio, 1966 : 145). Si les formes ont évolué, privilégiant à partir du VIIIe siècle les formes accusatives pour l'expression du datif, son caractère familier, déjà présent en latin, a été transmis sans aucun doute aux langues romanes tels le français et l'espagnol (Bassols, 1948). Bien que ce caractère familier le rende un élément plus naturel dans le discours oral, ce type de datif a été repris dans les textes littéraires, notamment français, comme un marqueur d'oralité dans le discours des personnages. Cependant, il nous intéresse de savoir dans quelle mesure ces documents écrits retracent l'usage naturel des locuteurs des deux langues ou s'il s'agit pour le contraire, d'un élément servant à recréer une oralité stéréotypée ayant pour but de distinguer les registres pouvant intervenir dans un texte littéraire. À une époque où oralité et écriture se retrouvent auprès des réseaux sociaux et d'autres moyens d'expression sur Internet, il nous intéresse de voir comment notre pronom d'étude, repris dans les textes écrits, notamment littéraires ${ }^{2}$ comme un signe d'oralité, s'utilise à l'écrit dans ce contexte " oralisant ". Partant donc ainsi d'un support écrit, nous cherchons la présence spontanée de cet outil afin de voir si son usage répond à la réalité des locuteurs, comme il en est question dans les textes littéraires où il est utilisé pour offrir une certaine vraisemblance aux discours tout en compensant leur caractère écrit. Or, nous considérons qu'une première définition théorique sur ce pronom, souvent délaissé dans

\footnotetext{
1 Notons par exemple la différence des verbes qui entraînent l'usage du pronom datif, que ce soit éthique ou argumental, comme nous pouvons apprécier dans le cas du verbe " obedecer ": en espagnol il est accompagné d'un complément d'objet direct, alors que son équivalent "obéir " entraîne en français un complément d'objet indirect : "Je lui obéit toujours ".

2 Nous avons constaté sa présence dans les parties dialoguées des ouvrages narratifs, tout comme dans les pièces de théâtre et les bandes dessinées en particulier.
} 
les grammaires, s'avère intéressante. Ainsi, nous proposerons une classification du pronom éthique dans les deux langues d'étude pour analyser ensuite quels sont ses implications et ses usages dans des corpus où oralité et écriture s'entrecroisent.

\section{LE DATIF ÉTHIQUE}

La définition du pronom éthique ne peut pas se passer de la délimitation des constructions verbales caractérisant le groupe des datifs auquel il appartient. De cette manière, nous estimons nécessaire de cerner ce type des constructions datives et les problèmes de classement repérés par plusieurs linguistes, avant de pouvoir définir grammaticalement le datif éthique.

\subsection{Constructions Datives}

La délimitation des structures verbales datives s'avère nécessaire pour aborder les particularités du datif éthique. Notons qu'à différence d'autres configurations verbales telles que le sujet ou encore le complément d'objet direct, comprises dans la construction verbale, le datif peut disparaître étant donné que sa présence n'est pas obligatoire pour la grammaticalité de nombreux verbes (Delbecque, 1992 : 125). Certains linguistes ont tenté d'établir, dans les deux langues, des classements afin de déterminer les cas où la construction dative se révèle nécessaire, conformant les " actants " de la phrase, contre les cas où cette construction peut être supprimée, les " circonstants " si nous tenons compte de la nomenclature établie par Tesnière (1959) ; or, et comme nous verrons par la suite, les conclusions ne sont pas toujours définitives. Une première classification détermine deux grands groupes qui acquièrent des appellatifs différents selon les auteurs. Leclère (1978) a établi une distinction dans les verbes ayant besoin du complément, étant donné que sa suppression peut entraîner un énoncé elliptique :

(1) a. Élisa a dit aux enfants de ne pas courir.

b. Elisa les $^{3}$ ha dicho a los niños que no corran.

(2) a. Élisa a dit de ne pas courir.

b. Elisa ha dicho que no corran.

Dans ces cas, le complément datif du verbe "dire " est sémantiquement nécessaire. Autrement dit, si le premier énoncé peut être considéré comme complet, dans (2) le complément indirect ("aux enfants") n'est pas explicite ; une transitivité invisible qui permet au complément, nécessaire dans la construction du verbe "dire "4 d'être sous-entendu de sorte que l'énoncé est compris comme elliptique. Or, il existe d'autres verbes dont le complément

\footnotetext{
${ }^{3}$ En espagnol, il est fréquent d'observer un dédoublement du complément datif indirect.

${ }^{4}$ Le verbe " dire " doit être classé comme trivalent si nous nous tenons à la nomenclature de Tesnière (1959).
} 
peut disparaitre sans que la phrase reste incomplète (exemples de Leclère, $1978: 66)$ :

(3) a. Paul a construit un jouet à Jean.

b. Paul le ha construido un juguete a Jean.

c. Paul a construit un jouet pour Jean.

d. Paul ha construido un juguete para Jean.

(4) a. Paul a construit un jouet.

b. Paul ha construido un juguete.

Contrairement à (2), (4) n'est pas elliptique dans la mesure où le verbe " construire " n'impose pas dans sa structure verbale l'existence d'un complément indirect. Les premiers exemples (1) et (2) se correspondent donc au groupe des datifs lexicaux, alors que lorsque le datif n'est pas déterminant dans la compréhension du verbe, Leclère le classe en tant que " datif nonlexical " ou "étendu ". De cette façon, le complément étendu présenté sous sa forme non-pronominale permet la commutation du syntagme " à N " par " pour N " lorsqu'il s'agit du bénéficiaire du procès (3c-d). Pour Leclère, ce dernier groupe de datif dit étendu ne pourrait pas être classé en tant qu'argument verbal car son usage n'est pas exigé par le verbe mais par l'usage des locuteurs. De son côté, le datif éthique, marquant " l'intéressement au procès " de la part du locuteur (Leclère, 1978 : 68), bien que proche du datif étendu, doit être distingué de ces deux groupes (Voir partie 2b).

Si cette distinction de groupes est assez homogène parmi les auteurs qui s'intéressent à l'étude du datif, les termes choisis par Leclère ont été largement mis en question. Roberge et Troberg (2007) les rejettent du fait de leur polysémie trompeuse tout en proposant deux nouveaux termes "complément thématique " vs " complément non-thématique ". D'ailleurs, le " nonthématique " est plus large et regroupe tous les datifs qui ne sont pas déterminés par le verbe : datif d'intérêt, datif sujet causatif, datif de possession et datif éthique. De son côté, Van Peteghem (2006) propose les termes " cas structuraux " vs " cas sémantiques "; et Barra Jover (1996) critique les limitations du classement de Leclère qui ne considère qu'un seul type de clitiques pour le datif étendu, alors que le datif éthique et le datif d'intérêt peuvent se présenter sous la forme de complément prépositionnel comme le remarquent Cano (1981) et Hernanz et Brucart (1987) : "se le rien, se le escapó y que, como muy bien señalan estos últimos, son "complementos preposicionales pronominalizables", ya que corresponden a frases como se ríen de ella o se escapó de él" (Barra Jover, 1996 : 124). Néanmoins, cette dernière remarque est discutée et Masullo (1992), repris par d'autres linguistes comme Cuervo (2003) ou encore Patriau (2007), affirme leur correction lorsque le datif n'appartient pas à la valence verbale et qu'il apparaît dans le discours à la suite d'une séparation dans l'intonation.

(5) a. El niño le estudió mucho // a María.

b. *L'enfant lui a beaucoup étudié // à Marie. 
Une casuistique reprise dans le cas du français comme nous pouvons observer dans les phrases suivantes. (Exemples de Kayne cité par Cuervo 2003 : 172).

(6) a. Elle lui a tué sa femme.

b. Ella le mató a su esposa.

(7) a. *Elle lui a tué sa femme à lui ${ }^{5}$.

b. Ella le mató su esposa a él (a Juan).

Dans le cas de l'espagnol, le classement du datif introduit des termes assez proches tels que "argumental" vs "no argumental" proposés par Gutiérrez (1999) ou encore "regido" vs "no regido". Ces derniers, ayant été introduits par Folgar, introduisent une nouvelle vision par laquelle une seule classe grammaticale est possible, conférant ainsi une importance notable au contexte extralinguistique dans l'interprétation des énoncés (Folgar cité par Barra Jover, 1996 : 122).

Une fois que nous avons établi le fonctionnement de ces constructions datives, nous pouvons nous interroger sur la définition du datif éthique dans nos deux langues d'étude. Un usage qui se caractérise notamment par son indépendance syntaxique.

\subsection{DÉlIMITATIONS DE L'USAGE DU DATIF ÉTHIQUE EN FRANÇAIS ET EN ESPAGNOL}

Malgré les différences structurales des deux langues, elles convergent dans l'usage des structures verbales qui leur sont propres et qui pourraient être définies globalement à partir du concept de valence verbale (Tesnière, 1959). Si cette définition est universelle (Coseriu, 1978), les actants des verbes dépendent de chaque langue comme le signalait le linguiste Gilbert Lazard (1994) dans L'Actance, ce qui explique la difficulté d'établir une équivalence entre les classements de deux langues différentes (Le Querler, 2012 : 176). Le datif éthique s'inscrit par conséquent dans un groupe grammatical dont les limites ont été discutées du fait de la complexité de cerner ces compléments accessoires et leur importance dans la phrase ${ }^{6}$.

\footnotetext{
${ }^{5}$ Cet énoncé que nous considérons agrammatical pourrait coexister également avec un énoncé grammatical lorsque le syntagme " à lui " acquiert une nuance emphatique. Dans cette interprétation grammaticale, le syntagme reprendrait emphatiquement le sens de possession "elle a tué sa femme ". Les deux énoncés peuvent donc coexister, mais il nous intéresse ici de signaler son caractère agrammatical si le sens n'est pas emphatique.
}

Elle lui a tué sa femme à lui VS *Elle lui a tué sa femme, à lui.

6 Il serait important d'ajouter d'ailleurs que le terme faisant référence à notre pronom d'étude s'avère
également assez problematique ; certains auteurs utilisent d'ailleurs plusieurs termes tels que datif
d'intérêt ou datif superflu pour le nommer (Patriau, 2007). Or, cette nomenclature pourrait être
confuse étant donné que les termes ne sont pas exactement équivalents. En effet, Bello (1847) définit
le datif superflu comme un type de datif n'étant pas nécessaire pour la réalisation verbale, proche
donc du datif d'intêrêt. Le datif éthique serait donc un sous-type de datif dans le groupe des datifs
superflus de Bello.
Cependant, nous éviterons le terme car il ne rend pas compte de l'importance que ce type de datif
possède à notre avis. D'autres nomenclatures sont celles de datif émotif et pronom d'intérêt explétif. 
écrit par la Real Academia de la Lengua Española comme un "pronombre dativo no requerido por el significado del verbo que se usa con intención afectiva para eludir a la persona que se ve afectada indirectamente por la acción verbal" (cfr. Diccionario de la Real Academia de la Lengua Española), ce pronom explétif utilisé normalement dans des discours familiers peut reprendre plusieurs personnes différentes, bien que tous les auteurs ne soient pas d'accord et que la délimitation reste problématique. Ainsi, des auteurs comme Delbecque en espagnol ou Deschamps en français font allusion au peu d'occurrences du pronom éthique à la deuxième personne du singulier tandis que Leclère (1976) relève l'ambiguïté de la structure de deuxième personne où le pronom pourrait être interprété comme éthique mais aussi comme une construction dative où le pronom servirait à reprendre le bénéficiaire de cet énoncé (8).

(8) a. Paul te fabriquera une table en vingt minutes (Exemple de Leclère, 1976 : 87)

b. Paul te hará una mesa en veinte minutos.

Quant à la troisième personne dont la représentation supposait moins de $18 \%$ dans le corpus analysé par Deschamps (2015), elle n'est pas conçue par certains linguistes comme Maldonado (1998), qui définit le datif éthique comme celui qui ne peut exister que dans les deux premières personnes. Gutiérrez Ordóñez (1999 : 1916) de son côté soutient l'existence du datif éthique sous la troisième personne : "Nada impide que el afectado por el proceso representado sea una tercera persona", bien que cette possibilité est moindre du fait de l'éloignement vis-à-vis du locuteur. Compte tenu des difficultés posées par ces contraintes morphologiques, nous nous sommes centrés dans cet article dans la définition du pronom de première personne, usage qui représenterait $80 \%$ des occurrences dans le cas du français selon Deschamps (2015) et qui reste également le plus naturel en espagnol. Dans le cas de l'espagnol, nous avons repéré aussi certaines occurrences concernant la première personne du pluriel que nous avons gardées dans notre corpus, car moins nombreuses que les pronoms singuliers, elles se rapportent à la première personne, la plus fréquente et moins problématique dans les deux langues. Or, sa délimitation en tant que pronom étant nécessaire pour cerner sa présence dans les contextes réels, il devient nécessaire de décrire les propriétés grammaticales qui le définissent dans nos deux langues d'étude.

Identifié également sous la nomenclature de datif émotif, ce pronom suppose au niveau sémantique une proximité relationnelle entre les locuteurs ou entre le locuteur et l'objet de la phrase, étant même ce rapport nécessaire pour l'acceptabilité de son usage (Patriau, 2007). Dans (8) l'interlocuteur comprend le rapport d'affectivité qui se crée entre le locuteur et le sujet de la phrase $^{7}$, condition fondamentale pour accepter l'énoncé. Cependant,

\footnotetext{
${ }^{7}$ Ce rapport peut se créer avec le sujet comme nous observons dans (8) ou avec un autre actant de la phrase (Cuervo, 2003), comme un complément d'objet direct ou un complément régime ou indirect, ce dernier n'ayant pas été registré dans notre corpus dans le cas du français.
} 
même si ce rapport d'affectivité peut être présent également dans les cas d'emploi de ce type de pronom en français (9), cette formulation ne serait pas correcte ; la proximité entre le locuteur et le sujet s'exprimerait par le biais d'un déterminant possessif, par exemple. Quoi qu'il en soit, ce rapport supposerait une justification syntaxique dans la mesure où il pourrait être interprété comme le possesseur de l'actant avec lequel ce rapport a été établi.

(9) a. Antonio se me fue a Alemania con la novia.

b. *Antoine m'est parti en Allemagne avec sa copine.

c. Mon Antoine est parti en Allemagne avec sa copine.

(10) a. Mais, qu'est-ce que vous me fichez-là ?

b. Pero, ¿qué me estáis haciendo?8

(11) a. Se me ha bebido cuatro botellas en cinco minutos.

b. Il m'a bu quatre bouteilles en cinq minutes.

Ce dernier exemple (11) pour le contraire, ne reprend pas le caractère affectif du pronom mais décrit un procès exceptionnel du point de vue du locuteur de sorte que l'interlocuteur est pris comme le témoin dudit procès 9 . Si l'implication sémantique du datif éthique est assez similaire dans les deux langues, Deschamps (2015 : 11) signale que dans certains cas ${ }^{10}$, il introduit dans la phrase une connotation négative : " [...] (menace, action violente, brimade, réprimande, acte d'autorité...) dirigée contre une personne physique ou morale (le complément animé) ". ${ }^{11}$ Il rassemble à titre d'exemple les prédicats avec lesquels apparaît cette expression du datif éthique :

Nettoyée à coups de bulldozer/noyée dans un chaudron d'acide/bouffée toute crue/envoyer chier le petit père/dynamitée dès 1905/envoyée bouder/donné à bouffer au crocodile/fait bouffer/fait valser d'un mur à l'autre/sodomisée à la batte/virée/déroulé l'intestin grêle/réduit en hachis/tiré dessus/fauché d'un coup de mon pilon/mettre en morceaux... (Deschamps, $2015: 11$ )

\footnotetext{
${ }^{8}$ Cet exemple reste douteux et ambigu car dans un contexte normal il serait interprété comme un complément essentiel compris dans la structure verbale : " hacer algo a alguien ". Or, il pourrait être aussi éthique dans des contextes concrets où le locuteur n'est pas le bénéficiaire de l'action mais exprime un rapport d'affectivité avec l'interlocuteur, de sorte que le pronom servirait à renforcer son implication par l'action des interlocuteurs. D'ailleurs, cette possible interprétation du pronom préverbal accompagné du verbe "hacer " pourrait se voir dans d'autres phrases ayant un sens similaire, comme dans : "No me hagas más tonterías " où le sens éthique est plus clair. Étant donné le changement de modalité qui rend plus clair l'exemple espagnol dans son sens datif, il faudrait se demander l'impact de l'usage de ce type de pronom explétif selon les modalités de la phrase. Ainsi, si nous observons que dans cet exemple, son usage dans le mode interrogatif en français est plus clair que son usage dans cette même modalité en espagnol, les exemples de datif dans les phrases impératives sont plus abondants dans les deux langues.

${ }_{9}$ Dans le cas de la phrase en français, cela pourrait être analysé comme un datif étendu où le sujet de la phrase aurait bu les quatre bouteilles du locuteur (Leclère, 1978).

10 Deschamps (2015) établit cette interprétation sémantique à partir d'un corpus sur lequel il a travaillé, et qui concerne notamment les cas d'accumulation de datifs.

${ }_{11}$ Que ce dernier soit grammaticalement présent dans la phrase comme sujet ou comme complément direct.
} 
Les linguistes espagnols qui ont abordé l'étude du datif éthique ne reprennent pas pourtant cette possibilité sémantique et d'ailleurs, nous pouvons remarquer, d'après les corpus travaillés, une différence de fréquence entre les cas où le datif entraîne des connotations violentes dans les exemples recueillis pour les deux langues ${ }^{12}$. Autrement dit, bien que nous attestions des usages présentant cette connotation en espagnol (12), leur apparition naturelle dans les discours des locuteurs est plus faible.

(12) "Al primer Bueno de Guzmán que entre, me lo destrozas a mordidas" (Benito Perez Galdós, Lo Prohibido, 1884).

Ainsi, ce type de pronom servant à manifester un "engagement dans le procès " (Leclère, 1976) que ce soit de la part du locuteur comme de celle de l'interlocuteur, adopte une forme identique à celle du pronom pré-verbal des autres datifs; si bien, il ne peut pas s'exprimer par un complément à $N$.

(13) a. Este niño no me come.

b. *Cet enfant ne me mange.

(13') a. *Este niño no come a mí.

b. *Cet enfant ne me mange à moi.

(14) a. Mets ton manteau, sinon tu vas me prendre froid !

b. ¡Ponte el abrigo que me vas a coger frio!

$\left(14^{\prime}\right)$ a. *Mets ton manteau, sinon tu vas prendre froid à ta mère !13

b. ¡Ponte el abrigo que le vas a coger frío a tu madre!

(15) a. Goûtez-moi ce gâteau, je veux savoir s'il est bon.

b. Pruébame esta tarta, a ver si está buena.

Ces exemples démontrent que dans les deux langues, bien que le datif soit exprimé par un pronom préverbal faisant référence à l'un des actants de la phrase, il ne peut apparaître que sous forme de pronom. Dans le cas $\mathrm{du}$ français, le datif peut adopter la forme du pronom tonique (15) pour les phrases impératives (Schön, 1997), qui sont d'ailleurs les plus fréquentes.

\footnotetext{
12 Ces connotations négatives associées à la violence ont été étudiées par des linguistes français tels que Deschamps (2015) qui en fait une classification sémantique et l'associe même à l'accumulation de pronoms éthiques ; cependant, cette possibilité sémantique n'a pas été abordé par les linguistes espagnols ayant traité le cas de ce pronom. En ce qui concerne les exemples en français, la plupart de ceux que nous avons développés dans notre corpus se caractérisent par les connotations sexuelles ou violentes. Si dans le cas de ce corpus, des exemples en espagnol de ce type n'ont pas été retrouvés, il en existe comme nous pouvons voir dans le corpus du datif éthique du Centre Virtuel Cervantes (cfr. https://cvc.cervantes.es/lengua/default.htm). Or, nous pouvons constater que d'un total de 73 exemples, seuls 4 ont des connotations de ce type. Et d'ailleurs, après avoir analysé ces exemples, nous pourrions dire même que l'usage du pronom éthique peut se rapporter dans certains cas non pas au procès violent en soi, mais à une relation affective avec celui qui va subir la torture et une souffrance de la part du locuteur : "Está de Dios - dije - que no me había de ir con el bayo. Hoy me lo cornean, ayer por poco no deja el cuero en el cangrejal", Ricardo Güiraldes, Don Segundo sombra (1926). 13 Ces énoncés (13') où le complément " à $\mathrm{N}$ " reprend le datif éthique, bien qu'étant agrammaticaux, pourraient à la limite être considérés comme acceptables dans certains contextes d'énonciations concrets, comme par exemple dans le cas d'une mère qui s'adresse à son enfant et se situe en tant que bénéficiaire de son propre énoncé ; ou encore, si c'est le père ou un parent qui le dit à l'enfant faisant de la mère par le rapport d'affectivité et son intérêt à préserver l'état de santé de son enfant, le bénéficiaire du message.
} 
Quant au niveau morphologique, il faudrait préciser que tout comme les pronoms préverbaux, les pronoms éthiques peuvent se décliner selon l'actant concerné et la personne grammaticale que cet actant représente ${ }^{14}$. Les formes plurielles quant à elles, bien que rares d'après Deschamps (2015 : 5), sont souvent accompagnées d'un autre clitique dont l'usage n'est pas obligatoire pour le verbe (16), c'est-à-dire, d'autres pronoms éthiques. Cette accumulation peu acceptée par les puristes, devient très caractéristique dans des usages où le procès expose un contexte belliqueux où le locuteur est impliqué violemment ${ }^{15}$ et n'a pas d'équivalence en espagnol (16b).

(16) a. "Je vais te me les disperser, moi ! ", Astérix et Obélix, Tome $7: 11$.

b. "Yo los dispersaré!16", Asterix y Obelix, Tomo 7: 11.

En effet, même dans les cas où trois pronoms clitiques coexistent, il n'y a qu'un seul pronom éthique étant les autres des manifestations des compléments nécessaires pour le verbe, ou bien des compléments liés à l'usage pronominal de certains verbes comme dans (17) où le complément argumental serait "lo" en espagnol et "le " en français et le pronom "te" de la phrase espagnole conformerait une marque de la pronominalisation du verbe "comer" en "comerse".

(17) a. No te me lo vayas a comer, que está malo.

b. Ne le mange pas, c'est mauvais.

Or, outre ces cas spécifiques d'accumulation, certains linguistes soutiennent que son usage doit être forcément accompagné d'un autre complément datif, ce dernier nécessaire dans la construction verbale : "Para que este elemento periférico pueda ocurrir es necesaria la presencia de otro clítico dativo en la misma estructura oracional" (Maldonado cité par Becerra, 2007 : 18). Tout de même, la liberté syntaxique de ce pronom qui échappe à la dépendance verbale, en français comme en espagnol, lui permet d'apparaitre malgré l'absence d'un autre pronom datif (18).

(18) a. Lucie est malade, elle m'a pris froid.

b. Lucía está mala, me ha cogido frío.

\footnotetext{
14 Toutefois, l'acceptation de certaines déclinaisons, notamment celles de deuxième et troisième personne est contestée par certains auteurs du fait de leur ambiguïté. Maldonado (1998) cité par Becerra (2007: 11) nie même l'existence d'un pronom éthique à la troisième personne : :Se trata de aquel dativo que solo puede ocurrir en las dos primeras personas". Un avis qui est partagé par la plupart des grammaires, comme nous pouvons observer dans celle de Riegel (1994: 226) : "Le datif éthique qui ne concerne que la deuxième personne ". En opposition avec ces affirmations, Gutiérrez Ordóñez (1999: 1916) affirme: «Nada impide que el afectado por el proceso representado sea una tercera persona".

15 Voir 3.

16 Traduction officielle. Nous pourrions contempler également de le traduire par : "Yo te los dispersaré». Dans ce cas-là, le pronom de deuxième personne ne serait pas analysé comme un pronom éthique car si le verbe est transitif direct, nous pourrions lui supposer un complément d'objet indirect : "dispersar algo a alguien". Cependant, ce dernier complément est souvent sous-entendu.
} 
Ainsi, le datif éthique ne pourrait pas être défini comme un sous-type de complément d'objet indirect. Pour Gómez Torrego dans la Nueva gramática de la lengua española. Morfologia y sintaxis (2009 : 309) le datif éthique conformerait l'un des sous-types d'un groupe indépendant de datifs qui serait plus vaste. Une affirmation qu'il faudrait revoir dans les recherches futures, mais qui nous éloigne maintenant de l'objectif de cet article : 1'usage du pronom éthique sur les réseaux sociaux.

\section{ENTRE ÉCRITURE ET ORALITÉ : LE CAS D'INTERNET ET DES RÉSEAUX SOCIAUX}

Le développement des nouvelles technologies de l'information et de la communication (TIC) lors de ces dernières années est à la base d'un retour à l'immédiat. L'accès rapide à un vaste ensemble de contenus tout comme la possibilité d'être en contact, d'une manière instantanée, avec des personnes ne partageant même pas l'espace géographique ont influencé la prolifération, lors de la dernière décennie, d'un nombre élevé de plateformes sur Internet comme les blogs, les forums de discussion ou les chats, ainsi que de nombreux réseaux sociaux à de multiples fins comme Youtube, Facebook, WhatsApp, Messenger, Instagram ou Twitter, entre autres. Ces applications permettent donc un accès soit à l'information, notamment audiovisuelle, soit à la communication, et partagent l'immédiat et la rapidité. Or, la rapidité offerte par cet essor technologique exige une écriture plus proche du registre oral, dans ce que certains chercheurs ont nommé comme "seconde oralité " à partir d'un parallèle avec les sociétés tribales où l'écrit ne l'emporte pas sur l'oral (J. Ong, 2002). L'écriture, considérée comme prestigieuse dans la conception grammaticale et de l'orthographe pour la standardisation des langues, se met au service de l'oral dans la conception de messages envoyés au sein de ces réseaux sociaux et des plateformes sur Internet, première activité des internautes, bien que pratiquée d'une manière et avec une fréquence différente en France et en Espagne :

$\mathrm{Al}$ analizar los tipos de actividades realizadas por Internet, se observa que tanto en hombres como en mujeres las actividades más realizadas son usar mensajería instantánea, intercambiar mensajes (por ejemplo, WhatsApp), buscar información sobre bienes y servicios, recibir o enviar correos electrónicos y ver contenidos de video de sitios para compartir (por ejemplo, YouTube). (INE, 2019).

L'usage des réseaux sociaux en Espagne, notamment WhatsApp qui reste leader avec 88\% des utilisateurs (cfr. Statista : https://es.statista.com/estadisticas /474955/redes-sociales-tasa-penetracion-usuarios-prevision-espana/), est en croissance et représente $85 \%$ de la population. La population française de son côté est moins active sur les médias sociaux avec 58,1\% d'utilisateurs actifs qui se servent notamment d'applications comme Facebook ou Messenger (cfr. Statista : https://fr.statista.com/statistiques/866846/taux-penetration-quotidien-reseaux-sociaux-par-plateforme-france/), WhatsApp restant à la traîne utilisée seulement par 31\% des usagers (Sara- 
gaglia, 2019). Dans l'ensemble, ces applications à but communicatif permettent donc un contact instantané dans la mesure où elles favorisent le développement des conversations écrites qui imitent le discours oral dans leur caractère immédiat tout comme dans leur rapprochement à la phonétique.

Notons d'ailleurs qu'avec l'incursion des messages vocaux, ou encore la reconnaissance vocale, il n'est même pas nécessaire de taper les messages. Bien que les différences entre écriture et oralité aient été traités d'une manière assez simpliste, comme le souligne Benveniste (1991), l'écrit ne présuppose pas toujours un style soigné, ni l'oral répond forcément au registre familier. Quoi qu'il en soit, une nouvelle catégorie de variation " oral/écrit " peut être considérée, tenant compte d'ailleurs, comme le développent Koch et Oesterreicher (1990 [2007]) de la possible influence du canal de communication dans la construction du discours.

Dans le cas de cet article, nous adhérons à la définition de Meschonnic par laquelle l'oralité est comprise comme "le rapport nécessaire, dans un discours, du primat rythmique et prosodique de son mode de signifier à ce que dit ce discours " (Meschonnic, 1983 : 280). Autrement dit, nous soutenons que le discours oral dans un contexte conversationnel, outre le fait d'exiger la réponse et l'interaction directe d'un interlocuteur, se caractérise par des aspects qui vont au-delà du signe linguistique, comme le débit de parole ou l'intonation qui peuvent changer complètement le sens d'une même phrase, tout comme un ensemble d'éléments pragmatiques et nonverbaux, à savoir les mimiques, les regards ou la posture entre autres. Il est évident que dans le contexte des réseaux sociaux, tous ces éléments externes à la langue ne sont pas présents, excepté le cas de l'intonation ou d'autres aspects prosodiques pour les messages vocaux. Mais, si ces éléments extralinguistiques ne peuvent pas être considérés dans les messages registrés dans les médias sociaux, il est intéressant de voir que les usagers y écrivent sans tenir compte de cette absence. De cette manière, nous pouvons constater que les textes, des fois abrégés phonétiquement, répondent à un code oralisé dont la compréhension est souvent associée aux éléments non-linguistiques qui devraient l'accompagner. En d'autres termes, ce type de service impose en raison du contact immédiat avec les interlocuteurs, un style d'écriture rapprochée d'un contexte où le canal de transmission serait oral.

Afin de cerner un corpus tenant compte de ces particularités stylistiques et qui puisse donc être pertinent pour notre étude, nous avons choisi d'une part des réseaux sociaux tels que WhatsApp, Facebook ou Instagram ${ }^{17}$ pour

\footnotetext{
17 Twitter, du fait de la limitation de caractères, a été exclu de notre analyse, étant d'ailleurs le cas de présence assez ambigu, comme par exemple dans : "PERO CHAVALES ME TENÉIS QUE CANTAR EL HIMNO BIEN EH A MÍ O ME CANTÁIS LOS PUEBLOS Y LA HUMANIDAD O NADA EH VAMO A HASÉ LAS COSA BIEN POR MI MARE YA HOME", où le "me" ne ferait pas partie de la structure "cantar algo a alguien" mais devrait être compris dans un sens datif servant à exprimer l'intérêt du locuteur sachant que l'hymne peut être chanté de deux manières différentes, à savoir "España" ou "los pueblos". Autrement dit, le locuteur de ce message comporte un intérêt particulier à ce que l'hymne soit chanté à "los pueblos" et non pas à "España", une implication qui est reprise par le pronom éthique.
} 
les deux langues, et d'autre part, des forums et des blogs sur Internet que nous avons repéré à l'aide du logiciel Sketch Engine. En effet, les commentaires retrouvés dans ces blogs reprennent le style qui nous intéresse dans notre recherche "d'oralité " dans l'écrit. Ainsi, ce logiciel nous a permis d'obtenir un ensemble de corpus en français et en espagnol, tout comme la possibilité de créer nos propres corpus à partir de la recherche de lemmes choisis dans un ensemble de sites web.

\subsection{LE CAS DU FRANÇAIS}

Pour l'analyse d'exemples en français, nous avons tenu compte d'une part des réseaux sociaux, où l'usage de ce pronom s'avère assez rare. Effectivement, sur un ensemble de 100 conversations sur WhatsApp, nous n'avons pu repérer qu'un seul exemple.

(19) Une autre perle/Toujours du même élève/Mon champion/Il m.en.a sorti 2.

(20) Regardez-moi cette photo digne des plus grands concours.

Le premier exemple (19) ne pouvant pas être considéré comme datif éthique, il renvoie à cette connotation sémantique d'appartenance de notre pronom d'étude. De notre côté, nous ne l'avons pas catalogué comme étendu car il peut être repris par la structure verbale ${ }^{18}$ : "quelqu'un sort quelque chose à quelqu'un ". Le besoin sémantique du complément bénéficiaire transforme l'interprétation du verbe " sortir ", qui ne serait donc pas intransitif, mais devrait être compris dans un sens rapproché de celui du verbe " dire ", et donc serait trivalent selon les catégories établies par Tesnière (1959). Le seul exemple que nous pouvons tirer (20), écrit par un locuteur parisien ${ }^{19}$ coïncide avec la forme morphologique la plus courante en français, à savoir le mode impératif. Cette application étant encore moins utilisée en France qu'en Espagne, notre corpus a été complété par Facebook tout comme son application Messenger. Les résultats restent cependant assez similaires et nous ne pouvons pas attester son usage.

Également, et étant donné qu'en français cerner son apparition est plus facile, nous avons utilisé le logiciel Sketch Engine afin de compiler notre corpus et de comptabiliser le nombre d'apparitions de chaque concordance. Or, l'extension de notre thème et la difficulté de cerner tous les usages du datif nous ont obligés à restreindre notre étude à des structures concrètes. Tout d'abord et comme nous avons développé plus haut, les confrontations concernant l'existence du datif éthique pour certaines personnes grammaticales comme la troisième personne, nous ont permis de restreindre notre corpus à la première personne dont l'usage est plus commun d'après les études de Deschamps (2015) et les cas comportant des ambiguités sont moindres. Ensuite, nous avons limité deux structures concrètes. D’une part, sachant que

\footnotetext{
${ }^{18} \mathrm{La}$ structure verbale de laquelle nous parlons serait conformée par les compléments indispensables du point de vue sémantique.

19 Il s'agit d'un locuteur d'entre 20 et 30 ans ayant des études supérieures.
} 
le datif éthique est normalement employé sous la forme des pronoms toniques pour le mode impératif, nous avons tenu compte du verbe " regarder " accompagné du pronom " me " et suivi d'un groupe nominal. En effet, cette duplication nominale suppose l'exclusion du pronom de la structure verbale et par conséquent sa définition en tant que pronom éthique.

\begin{tabular}{|c|c|c|}
\hline $\begin{array}{c}\text { Concordance de } \\
\text { recherche }\end{array}$ & Occurrences & Exemples \\
\hline "Regarde-moi ça" & 145 & $\begin{array}{l}\text { "Ahahaha Regarde-moi ça! Elle s'est crue où ? } \\
\text { On fait pas de la breakdance (forumgratuit.fr) } \\
\text { "Elle ne lâchait pas Tom du regard. Regarde-moi } \\
\text { ça... il est tellement mignon (forumpro.fr) } \\
\text { " Regarde-moi ça, c'est un vrai désastre ! " (free.fr) } \\
\text { " Regarde-moi ça! Elle s'est crue où ? On fait pas } \\
\text { de la breakdance ici " (forumgratuit.net) }\end{array}$ \\
\hline $\begin{array}{l}\text { "Regarde-moi } \\
\text { ce/cette/ces" }\end{array}$ & 265 & $\begin{array}{c}\text { "Regarde-moi ce gros débile qui ne ressemble à } \\
\text { rien " (free.fr) } \\
\text { " Hey mon ami ! Regarde-moi cette femelle de ser- } \\
\text { veuse !" (videonues.com) } \\
\text { "Regarde-moi ces bras qu'elles ont, on croirait des } \\
\text { allumettes" (bonbonze.net) } \\
\text { "Regarde-moi ces cons, je les arnaque et ils en } \\
\text { veulent encore... " (seneweb.com) }\end{array}$ \\
\hline "Regardez-moi ça " & 290 & $\begin{array}{c}\text { "[elles] s'amusent comme des... À LUI SUCER LA } \\
\text { BITE... Regardez-moi ça, elles sont deux à jouer } \\
\text { avec ses couilles... " (porno-streaming.net) } \\
\text { " Mais, regardez-moi ça! Une vache sur un fau- } \\
\text { teuil roulant! " (kiweo.com) } \\
\text { "Regardez-moi ça, je pense que mon tee-shirt est } \\
\text { vraiment foutu !" (berkeley-u.com) } \\
\text { " Regardez-moi ça, le fils de pute qui magouille } \\
\text { avec Pasqua !" (ab-ante.com) }\end{array}$ \\
\hline $\begin{array}{l}\text { "Regardez-moi } \\
\text { ce/cette/ces" }\end{array}$ & 817 & $\begin{array}{l}\text { "Tout le monde peut se taper cette nymphomane. } \\
\text { Regardez-moi ce vieux cochon qui lui lèche la } \\
\text { chatte " (films-de-cul.com) } \\
\text { " Regardez-moi cette cochonne blonde ultra ban- } \\
\text { dante ? " (video-film-porno.com) } \\
\text { " Regardez-moi cette gonzesse de } 18 \text { ans complète- } \\
\text { ment indécente " (filmsdesexe.net) } \\
\text { "Tout le monde va aimer ce visage, mais regardez- } \\
\text { moi ce cul, regardez-moi ces seins... " (films-de- } \\
\text { culs.com) }\end{array}$ \\
\hline
\end{tabular}

Tableau 1 : Apparition du datif éthique dans les constructions " regarde(z)-moi ça "à partir du logiciel Scketch Engine

D’autre part, il faut savoir que le pronom éthique est souvent accompagné d'autres pronoms qui peuvent être nécessaires pour le verbe, ou d'autres éthiques. Cette dernière possibilité, plus commune en français qu'en espagnol, a été étudié par Deschamps (2015) dans ce qu'il nomme " les accumulations ". Or, le pronom éthique peut accompagner également des compléments essentiels respectant dans ce cas-là l'ordre naturel de la complémentation en français, à savoir : Sujet > COI > Pronom éthique $>$ COD > Verbe. Cependant, il faut préciser que si cerner les structures propices à l'apparition de ce type de pronom est plus facile en français, le comptage d'occurrences a été plus complexe et reste approximatif du fait que de nombreux exemples en font un usage qui ne relève pas du datif éthique mais d'incorrections linguistiques. De cette manière, nous pouvons apprécier par 
exemple que pour le groupe " te me le " le premier pronom devrait se correspondre dans certains cas (21) au pronom personnel " tu ". De même, ce problème a été repéré dans les cas de " te nous " (22) et " te me "20 (23).

(21) "Je veux bien y croire... et j'ai hâte que te me le prouves " (berkeley-u.com). (22) "Puis déjà te nous connais en vrai Sev et moi " (canalblog.com).

(23) "Tu m'embrasses, puis te me repousses et finalement, tu m’ignores " (free.fr).

\begin{tabular}{|c|c|c|}
\hline Concordance de recherche & Occurrences & Exemple \\
\hline "Te me le" & 52 & $\begin{array}{l}\text { "Je vais te me le foutre dans l'arène avec } \\
\text { les loups cet espèce d'imbécile " (20mi- } \\
\text { nutes-blogs.fr) } \\
\text { "M'vais te me le lui balancer une grenade à } \\
\text { la bouse de vache " (info.com) } \\
\text { " Allez, je vais te me le faire décoller ce } \\
\text { fucking plane!" (free.fr) }\end{array}$ \\
\hline "Te me la" & 16 & $\begin{array}{c}\text { "Préviens, faites pas chier les gars, moi je } \\
\text { vais te me la savourer cette première jour- } \\
\text { née " (akynou.fr) } \\
\text { " je te creuse ! Et je te la dilate la jugeote ! } \\
\text { Et je te me la tyrannise ! " (garsbyon- } \\
\text { line.eu) } \\
\text { "Salut les gens, comment que j'vais te me } \\
\text { la torcher vite fait la journée au taf au- } \\
\text { jourd'hui " (forumactif.com) }\end{array}$ \\
\hline "Te me les" & 19 & $\begin{array}{l}\text { "Oui, c'est vrai. Quand à ces créatures, je } \\
\text { vais te me les massacrer sans lever le petit } \\
\text { doigt, tu vas voir " (ratchet-galaxy.com) } \\
\text { " (cette fois c'est les européennes) : il va te } \\
\text { me les nettoyer, les banlieues ! " (tdg.ch) } \\
\text { "Après ça, les termites, je te me les prends } \\
\text { une par une " (berlol.net) }\end{array}$ \\
\hline "Me te le/la" & 6 & $\begin{array}{c}\text { "Je trouve un homme pareil dans les buis- } \\
\text { sons, je me te le viole sur place !!! (lol)" } \\
\text { (pierre-de-tear.com) } \\
\text { " j'peux pas accuser j'ai pas vu mais si ja- } \\
\text { mais je me te le chope sur la fête ????? } \\
\text { (heu pardon sur le fait) " (donjons-tre- } \\
\text { sors.com) }\end{array}$ \\
\hline "Te nous" & 160 & $\begin{array}{l}\text { "J'ai lu ce besoin de te nous pomper de } \\
\text { fric " (cree-mon-blog.info) } \\
\text { " Plus le temps passait, plus elle te nous } \\
\text { sortait des discours de grosse droitasse " } \\
\text { (zeugmas.com) } \\
\text { " Que dieu (ALLAH) te nous protège Amine. } \\
\text { Salam. Bon courage ma sœur " (iman- } \\
\text { way.com) }\end{array}$ \\
\hline "Te me" & 432 & $\begin{array}{c}\text { "Je vais te me griller ce connard à jamais } \\
\text { de la fête " (realbb.net) }\end{array}$ \\
\hline
\end{tabular}

Tableau 2 : Apparition du datif éthique dans les constructions " te me " à partir du logiciel Scketch Engine

\footnotetext{
20 Plusieurs exemples tirés du corpus peuvent nous permettre de nous interroger également de l'usage de cette expression comme caractéristique d'une variété diatopique dans la mesure où plusieurs exemples ont été tirés de sites canadiens par exemple, ce que nous pouvons apprécier également dans certains éléments morphosyntaxiques de ces phrases, outre l'emploi du groupe " te me ": "Dans le temps, quand nous avions fini de manger, te me caressais le menton " (allmyblog.com).
} 
Outre certaines fautes, comme l'emploi du "quand " pour la locution " quant ", nous considérons que ces textes écrits font preuve d'un caractère oral dans la mesure où ils ont été créés spontanément, bien qu'ils aient laissé une trace sur Internet, ce que nous voyons dans certains éléments emphatiques tout comme dans l'absence de la première particule négative. En ce qui concerne le datif éthique, s'il incarnait un rapport spécifique entre locuteur et objet de l'énoncé de caractère émotif ou affectif, nous pouvons noter dans les exemples du tableau, représentatifs pour la plupart des énoncés conformant notre corpus, ce qu'avait relevé Deschamps (2015) au sujet des accumulations et de leur caractère violent (24) et sexuel (25).

(24) "Je vais te me griller ce connard à jamais de la fête " (realbb.net).

(25) " Elle a la chatte qui déborde de bonheur. Regarde-moi ce chef-d'œuvre ! " (sexeet-videos.net).

\subsection{LE CAS DE L'ESPAGNOL}

L'analyse du corpus espagnol a été plus productive car pour notre ensemble de 100 conversations enregistrées sur l'application WhatsApp ${ }^{21}$, nous avons repéré $20^{22}$ occurrences du datif éthique, si bien il y a des cas où cette étiquette s'avère ambiguë. Quant aux locuteurs, aspect que nous estimons important pour la description d'apparition de ce pronom, nous pouvons observer que la plupart sont des femmes âgées de plus de 25 ans ; quoique quatre locuteurs sont masculins, âgés d'entre 22 et 60 ans. Pour la plupart, les locuteurs des conversations concernées par l'usage du datif sont d'origine andalouse, mais d'autres origines ont été également enregistrées, à savoir Cuenca, Burgos et Madrid. Étant donné que les conversations ont été tirées d'une application de messagerie instantanée, elles appartiennent toutes à un registre familier et expriment notamment un rapport d'intérêt ou d'affectivité entre le locuteur et l'interlocuteur (26), ou l'objet personnel de la phrase (27). Enfin, ce rapport est à la base d'une certaine possession affective ressentie par le locuteur. Par exemple, nous observons dans (28) ce sentiment par lequel le locuteur utilise le pronom pour faire référence à ses élèves. En (29), le rapport est clair aussi dans la mesure où le locuteur et le sujet de la phrase sont conjoints.

(26) «Si tenéis hambre cenad, vale? Y ducháos. No me seáis guarretes».

(27) «No le ha vuelto a dar más fiebre se le escucha la voz tomada, pero bueno, la he llevado porque me da pena que se le junten todos los exámenes, que hoy tenía 2 así que!! Espero que no me recaiga".

(28) "Lo acabo de mirar y se me han inscrito 4 más".

(29) "Que dice Victor que el se apunta a cantar ranchera!! Mira que preparao se me ha puesto!!!".

\footnotetext{
21 Nous n'avons tenu compte que des messages écrits, les enregistrements vocaux ont été supprimés de notre corpus ne faisant pas partie du niveau écrit.

22 Nous avons respecté dans nos exemples le texte des locuteurs qui ont été retranscrits sans aucune modification de notre part, les fautes d'orthographe faisant donc partie des messages.
} 
L’intérêt du locuteur par le procès demandé à son interlocuteur est également exprimé par le datif éthique dans deux exemples du corpus :

(30) "acuerdate de traerme el libro mañana por favor es vital no te me vayas a olvidar que me va a dar algo con tanto agobiOooOooooo".

(31) "La última oportunidad!! Porfi compartirme ${ }^{23}$ la info !!».

Or, si le pronom peut être classé sans problème dans les énoncés précédents, il faudrait noter un cas d'ambiguité dans (32). En effet, que le pronom soit considéré comme datif éthique ou comme objet indirect dépend de l'antécédent que nous lui accordons. Si le "me" reprend la joie que le locuteur ressent suite à l'investiture du gouvernement, il ne pourrait pas être compris comme datif car il ferait partie de la structure verbale. Cependant, si le pronom fait allusion au procès d'investiture, ce qui est le plus probable d'après le contexte, il s'agit d'un pronom éthique dans la mesure où il exprime l'implication émotionnelle du locuteur dans ce procès et qu'il peut être supprimé sans que cela nuise le sens de la phrase ${ }^{24}$.

(32) «Estoy very happy después de esta investidura tan agónica. Espero que me dure please please pleasen.

Outre l'application WhatsApp, nous avons considéré d'autres réseaux sociaux sur lesquels les locuteurs s'expriment d'une manière directe qui relève aussi de l'oralité, permettant d'interagir avec d'autres utilisateurs à travers les commentaires, les messageries ou encore les réactions. D'un côté, en ce qui concerne l'application Facebook qui permet de partager des opinions, des évènements et des messages viraux ${ }^{25}$, nous avons enregistré dans notre corpus les exemples produits par les utilisateurs dont l'usage du datif éthique se rapporte encore une fois à l'implication émotive du locuteur. Pour ce qui est des énoncés (34) et (35) il faut noter qu'ils reprennent une situation fictive pour annoncer une pièce théâtrale ; or les liens de parenté et le rapport émotionnel entre les personnages de la pièce, ou encore entre le locuteur et les personnages se rapprochent d'un usage courant du datif éthique en sorte que l'énoncé semble plus naturel aux yeux des récepteurs du message. Autrement dit, le pronom participe au jeu par lequel les possibles spectateurs deviennent les proches des mariés :

(33) «Como nos ha crecido!! que pare el tiempo... que en nada se nos independiza!!».

\footnotetext{
23 Cette déformation de l'impératif qui est exprimée par le biais d'un infinitif auquel le locuteur a ajouté le pronom éthique est très courante dans le registre familier et fait preuve par conséquent de la nature de cette conversation.

${ }^{24}$ Le sens resterait le même mais la force serait moindre du fait que si le pronom peut être considéré comme superflu au niveau sémantique son usage répond toujours à une implication qui se perd avec sa suppression.

25 Nous avons repéré un message viral qui circule sur Facebook mais qui a été écarté du corpus du fait qu'il appartient à l'espagnol parlé en Amérique et qu'il faudrait aborder séparément : "Envidio a la gente que saca a pasear al perro sin correa y el perro hace el recorrido todo prolijito. Yo suelto a mi perro sin correa y el cabrón se come a 4 palomas, lo muerden 3 perros y seguro casi me lo atropellan. Y yo atrás persiguiendolo como un tonto".
} 
(34) "Ay, qué nervios! Que en unas horas se me casa la nena".

(35) «Este año nos vamos de boda!! El Tenorio se nos casa...o eso es lo que parece... ¿llegará al altar?".

(36) "Me caen bien esas personas que te ven comiendo y no te piden, y uno les ofrece y no aceptan. Dios me los bendiga".

(37) "Como se quieren mis principes. Se me hacen mayores, pero siguen siendo fiel al amor que nos dan cada segundo de sus vidas".

D'un autre côté, Instagram, l'autre réseau social que nous avons considéré pour notre étude, bien que plus éloigné des rapports directs que Facebook ou WhatsApp, peut entraîner des fois un langage proche de la langue parlée, notamment pour les stories du fait de leur spontanéité. Ainsi, nous avons trouvé deux exemples où nous observons encore la proximité relationnelle qui se voit renforcée par la présence du pronom éthique :

(38) "Se me va para que me eche mejor la siesta".

(39) "Eres imprescindible compi, no te me vayas nunca. Felicidades bro, te quiero mucho. Nos vemos pronto".

Enfin, et comme pour le cas du français, nous avons créé un corpus tiré de blogs et des sites internet permettant l'accès direct des utilisateurs à l'aide du logiciel Sketch Engine. Cependant, les difficultés pour cerner le pronom éthique espagnol ont restreint cette recherche, de sorte qu'il s'avère très difficile de comptabiliser les cas réels. Ainsi, pour des occurrences comme "mírame esto" et ses dérivés, nous n'avons pas obtenu de résultats ; mais pour le groupe " te me ", où le "me " peut être associé à un pronom éthique, le logiciel nous renvoie 6593 occurrences. Ces résultats nous mènent à erreur, car le programme signale aussi les verbes pronominaux ou encore d'autres cas qui échappent au pronom éthique. La délimitation de ce pronom dans le cas de l'espagnol s'avère impossible.

(40) "Me vuelves a contar lo mismo cuando los talibanes atenten en España por nuestra implicacion en Afganistan majete y mientras tanto te me vas a tomar por el culo" (abc.es).

(41) "No te me desanimes, eh? Los días malos los tenemos, pero poco a poco aprendemos a ser fuertes" (foroadelgazar.es).

(42) "Así que tú descansa y mañana el médico ya te dirá, ok? pero no te me pongas histérica eh!!" (glamour.es).

(43) «Las quiero muchísimoo! Mi niñaa pero como que te me vas?? porke?? no te vayas jooooo" (netmoms.es).

(44) «ja, ja...abu Alice donde andabas preciosa, no te me pierdas, hoy pega hablar de recetas por lo visto" (verli.es).

(45) "Nada Fiestero que no nos entendemos, que te me vas por los cerros de Úbeda..." (ibaneza.es).

(46) «No te me enfades Marío eh, que sabes que te apreciamos» (behcet.es).

Ces exemples, assez représentatifs du corpus, reprennent une fois de plus les mêmes rapports observés dans les exemples précédents où le datif 
thique exprime une relation affective de la part du locuteur. Mais, contrairement aux cas précédents, nous retrouvons ici une occurrence au caractère agressif (40), un autre usage propre à ce type de pronom. Un domaine qui resterait ouvert pour des recherches futures étant donné que nous nous sommes limités aux implications émotionnelles plus présentes dans le corpus espagnol.

\section{EN GUISE DE CONCLUSION}

Souvent relégué à un second plan et considéré comme superflu du fait de ne pas être nécessaire à la réalisation verbale, le pronom éthique reste fondamental au niveau de l'énonciation, malgré son difficile classement syntaxique. Ainsi, repris en littérature pour caractériser le " parler " au niveau écrit, nous avons pu vérifier que son usage est réel et se manifeste même dans des contextes limitrophes entre écriture et oralité, comme il arrive dans le cas des réseaux sociaux. L'importance dudit pronom se joue donc dans son usage malgré le fait que les conversations écrites se passent de certaines normes d'orthographe visant à raccourcir le temps d'écriture. En effet, si ce pronom considéré comme " superflu " n'est pas supprimé dans ce contexte, ce n'est que par son importance au niveau sémantique. En même temps, ces usages font preuve de sa présence au niveau oral dans les deux langues de notre étude.

Force est de constater cependant les différences qui se créent dans l'usage du pronom en français et en espagnol. Les corpus analysés laissent voir comment le pronom est beaucoup moins utilisé dans les contextes francophones qu'hispanophones. De la même manière, les valeurs sémantiques sont distinctes : si les cas français s'ouvrent plutôt vers les procès extraordinaires ou expriment des rapports de violence et un intérêt sexuel à caractère détrimentaire, notamment sur les commentaires répertoriés sur Internet ; en espagnol, la valeur belliqueuse est moins présente et les liens d'affectivité, voire de possession prédominent dans les corpus. Une conclusion qui n'est que provisoire car la recherche est encore en cours et les résultats pourront varier une fois que nous aurons élargi nos corpus.

\section{REFERENCIAS}

BARRA JOVER, M. (1996): "Dativo de interés, dativo aspectual y las marcas de aspecto perfectivo en español", Verba: Anuario galego de filoxofia [En ligne], 23, pp. 121-146. [http://hdl.handle.net/10347/32 68] (Consulté le 10 septembre 2019).

BASSOLS DE CLIMENT, M. (1948): «Sintaxis histórica de la lengua latina", II, 1. Las formas personales del verbo. Barcelona, CSIC - Publicaciones de la Escuela de Filología.

BENVENISTE, C. B. (1991) : "Les études sur l'oral et le travail d'écriture de certains poètes contemporain ",

Langue Française, 89. [https://www.persee.fr/doc/lfr_0023-

8368_1991_num_89_1_5763]

(Consulté le 12 novembre 2019). 
CANO, R. (1981): Estructuras sintácticas transitivas en el español actual, Madrid: Gredos.

COSERIU, E. (1978): "Los universales del lenguaje (y los otros)", Estudios de lingüística funcional. pp. 148-205. Madrid: Gredos.

CUERVO, C. (2003): "La alternancia causativa y su interacción con argumentos dativos", Revista de Linguïstica Teórica y Aplicada, 46(1), pp. 55-79.

DELBECQUE, N. (1984): «El Dativo español: Una tipología", Villegas, J. (ed.), Actas del XI Congreso de la Asociación Internacional de Hispanistas, Louvain: Katholieke Universiteit Leuven, 1, pp. 124-138.

DESCHAMPS, A. (2015) : " Je vais te me lui régler son compte : les limites de la syntaxe", Pratiques [En ligne], pp. 167-168. [http:// pratiques.revues.org/2687] (Consulté le 7 septembre 2019).

DICCIONARIO DE LA RAE (2019): [https://dle.rae.es/].

GUTIÉRREZ ORDÓÑEZ, S. (1999): "Los dativos", Bosque, I. et Demonte, V. (éds.) Gramática descriptiva de la lengua española. Madrid: Real Academia Española, pp. 18551930.

GUTIÉRREZ ORDÓÑEZ， S. (1977): "Sobre los dativos superfluos", Archivum, pp. 27-28. [https://www.unioviedo.es/reuni do/index.php/RFF/article/view/ 2068] (Consulté le 27 août 2019).

HERNANZ, M. L., BRUCART, J. M. (1987): La sintaxis. Barcelona: Crítica.

INE (2019): "Población que usa Internet (en los últimos tres meses). Tipo de actividades realizadas por Internet", Servicio de Publicaciones Instituto Nacional de Estadística. [https://www.ine.es/ss/Satellite?L $=$ es_ES\&c=INESeccion_C\&cid $=1259925528782 \& \mathrm{p}=$ 1254735110672\&pagename=ProductosYServicios\%2FPYSLayout] (Consulté le 20 novembre 2019).

KOCH, P. \& OESTERREICHER, W. (1990 [2007]): Pragmatics, Cambridge: Cambridge University Press.
LAZARD, G. (1994) : L'Actance. Paris : PUF.

LECLĖRE, C. (1976) : " Datifs syntaxiques et datif éthique " dans Chevalier, J. et Gross, M.(éds) Méthodes en grammaire française, Paris : Klincksieck, pp. 73-96.

LECLĖRE, C. (1978) : "Sur une classe de verbes datifs ", Langue française, 39. Études de syntaxe française [En ligne], pp. 66-75. [http://www.persee.fr/doc/1fr_00238368_1978_num_39_1_6128] (Consulté le 7 juillet 2019).

LE QUERLER, N. (2012) : "Valence et complémentation : l'exemple des verbes trivalents en francais contemporain ", Annales de Normandie, 2012/2 [En ligne], pp. 175188 [https://www.cairn.info/revue-annales-de-normandie-20122-page-175.htm] (Consulté le 6 septembre 2018).

MALDONADO, R. (1998): A media voz. Problemas conceptuales del clítico "Se". Mexico: UNAM.

MASULLO, P. J. (1992): Incorporation and case theory in Spanish. A crosslinguistic perspective. Thèse doctorale en linguistique. Seattle: University of Washington.

MESCHONNIC, H. (1983) : Critique du rythme. Lagrasse: Verdier.

ONG, W. J. (2002 [1982]): Orality and Literacy: The Technologizing of the Word, New York: Routledge.

PATRIAU HILDEBRANDT, C. A. (2007): Semántica y sintaxis de los dativos de interés del castellano, Lima: Pontificia Universidad Católica del Perú.

RAE \& ASOCIACIÓN DE ACADEMIAS DE LA LENGUA ESPAÑOLA (2009): Nueva gramática de la lengua española. Morfología y sintaxis. Madrid: Espasa.

RIEGEL, M. (2016) : " Le Groupe Verbal ", Riegel, M., Pellat J. C. et Rioul, R., Grammaire Méthodique du français. Paris : Presses Universitaires de France, pp. 215-241.

ROBERGE, Y. \& TROBERG, M. (2007) : "Les objets indirects non thématiques en français ", Radišić, M. : 
Actes du congrès annuel de l'Association canadienne de linguistique, Toronto : Université de Toronto, pp. 1-15.

RUBIO, L. (1966): "Introducción a la sintaxis estructural del latín", Casos y preposiciones, 1, Barcelona, Ariel.

SARAGAGLIA, B. (2019) : " E-commerce, réseaux sociaux... le comportement des internautes français en chiffres ", Capital. [https://www.capital.fr/-economiepolitique/e-commerce-reseauxsociaux-le-comportement-desinternautes-francais-en-chiffres1348066] (Consulté le 20 novembre 2019).

SCHÖN, J. (1997) : " De ce qui, dans le dialogue, défie la grammaire : l'exemple du datif ", dans
Weigand, E. et Hauenherm, E., Analysis : Units, relations and strategies beyond the sentence, Berlin : De Gruyter, pp. 195206. [http://ebookcentral.proquest.com/lib/bibucascbebooks/detail.action? doclD=3041214] (Consulté le 7 août 2019).

TESNIĖRE, L. (1959) : Éléments de syntaxe structurale, Paris : Klincksieck.

VAN PETEGHEM, M. (2006) : " Le datif en français : un cas structural ", Journal of French langage Studies [En ligne], 16, pp. 93-110. [http://www.academia.edu/7003430/Le_datif_en_français_un_cas_structural] (Consulté en ligne le 21 septembre 2019). 